surfen reduces neuroinflammation and cellular infiltration in EAE, some families of proteoglycans such as heparan sulfate proteoglycans may serve to promote remyelination centrally where general antagonism should be avoided.

CONFLicts OF INTEREST:

None.

\section{Abstract A7}

EphrinB3 and EphrinB4 Receptors are potential therapeutic targets in glioblastoma

R. Sreeraman Kumar ${ }^{1}$, R.J.B. Macaulay ${ }^{2}$, H.C. Rutherford ${ }^{2}$, N. Barkey ${ }^{3}$, J. Koomen ${ }^{4}$, D.L. Morse ${ }^{3}$

${ }^{1}$ Departments of Radiation Oncology; ${ }^{2}$ Anatomic Pathology;

${ }^{3}$ Cancer Imaging and Metabolism, and; ${ }^{4}$ Molecular Oncology \& Proteomics Core, H.L. Moffitt Cancer Center, Tampa, FL

doi:10.1017/cjn.2015.373

Glioblastoma (GBM) is the most common malignant neoplasm of the central nervous system in adults. Despite advances in surgery, chemotherapy, and radiation technique, median overall survival remains dismal at 5 years after diagnosis. CD133 is a known putative cancer stem cell marker, and we aimed to identify markers in CD133 + GBM tumour initiating cell lines (TICs) with an infiltrative phenotype that could serve as therapeutic targets.

Expression (mRNA) microarray datasets including 22,278 probes for known cell-surface markers in three CD133 + TICs and 17 normal brain tissue lines were obtained. By expression profiling, we identified genes with uniformly high mRNA expression, filtered for known localization at the cell-surface, and for non- or low expression in normal brain; amongst the highly expressed were ephrin B3 receptor (EphB3), ephrin B4 receptor (EphB4) and fibroblast growth factor receptor (FGFR1). Protein expression was established by mass spectrometry using CD133+ cell line extracts. These were further evaluated in 27 patient GBM (IDH-wildtype) tumour samples by immunohistochemistry, both in the tumour and at the brain-tumor interface. Expression in $>50 \%$ of tumour cells was enumerated as 7/27 for EphB3, 8/27 for EphB4, and 18/27 for FGFR1. Most tumours failed to exhibit a gradient of expression across the brain-tumor interface. Expression was occasionally noted in normal-appearing cells, particularly pyramidal neurons; most reactive-appearing astrocytes also strongly expressed FGFR1. Correlation with clinical parameters may disclose subsets of these tumours with varying infiltrative potential.

CONFLicts OF INTEREST:

None.

\section{Abstract A8}

Precision Care of Brain Tumour Patients via Personalized OncoGenomics - Promises and Challenges

\section{S. Yip, M.A. Marra}

Genome Sciences Centre, BC Cancer Agency, BC, Canada

doi:10.1017/cjn.2015.374
Cancerous behaviour is invariably governed by abnormalities within the genomes and epigenomes of tumour cells. These include mutations, copy number alterations such as amplifications, and structural variations that translate into aberrant transcripts and ultimately proteins. Examples of such changes in glioma include IDH1 R132H mutation, EGFR amplification, and FGFR3-TACC3 fusions. However, there are myriad other changes that could alter the biology of tumour with prognostic significance. Some, such as the mTOR pathway, maybe targeted by molecular therapeutic agents.

The BCCA Personalized OncoGenomics (POG) initiative utilizes whole genome, transcriptome, and panel sequencing of tumour to identify aberrant pathways for potential therapeutic intervention. POG aims to return informative and potentially actionable results within five to six weeks from time of biopsy. Over 250 patients have been sequenced in the past two years including 7 patients with CNS malignancies. In all cases, the data generated has served to confirm or re-align pathological diagnoses and also to identify aberrant genes, transcripts, and cellular pathways. In our hands, the integration of transcriptome and genome data has proven to be invaluable especially in comparing global gene expression profile against public cancer databases such as TCGA and ICGA. Moreover, we have incorporated analytic pipeline for measuring the expression of immune-related genes such as PDL1. Lastly, we are analyzing the mutational load and context to derive a "signature" which may inform on the molecular causation of the tumour. POG generates multilayer and granular genomic data that may provide clinical insight and treatment options for different tumour groups.

CONFLiCTS OF INTEREST:

None.

\section{Abstract A9}

\section{Incomplete seizure reduction following resection of focal} oligodendroglial hyperplasia

J.A.E. Radic ${ }^{1}$, H.C. Rutherford ${ }^{2}$, R.J.B. Macaulay ${ }^{2}$, R.M. Sadler ${ }^{3}$, T. Bardouille ${ }^{4}$, A. Ross ${ }^{5}$, D.B. Clarke ${ }^{1}$

${ }^{1}$ Divisions of Neurosurgery and; ${ }^{2}$ Department of Anatomic Pathology, Moffitt Cancer Center, University of South Florida, Tampa, FL, USA; ${ }^{3}$ Neurology, Dalhousie University, Halifax, NS, Canada; ${ }^{4}$ Biomedical Translational Imaging Centre, IWK Health Centre, Halifax, NS, Canada, and; ${ }^{5}$ Division of Nuclear Medicine, Department of Diagnostic Radiology, Dalhousie University, Halifax, NS, Canada

doi:10.1017/cjn.2015.375

The patient presented as a 39 year old right hand dominant female with medically refractory epilepsy. Pre-operative investigations, including MRI, electro- and magneto-encephalography, and fluorodeoxyglucose positron emission tomography (PET) were concordant with a lesion in the right inferomesial frontal lobe. Subdural electrode recordings demonstrated seizure onset in the right anterior inferior frontal lobe. The patient underwent surgical resection of the lesion. 